\title{
Phylogenomic analysis of Lake Malawi cichlid fishes: Further evidence that the three-stage model of diversification does not fit
}

\author{
Christopher Darrin Hulsey ${ }^{\mathrm{a}, *}$, Jimmy Zheng $^{\mathrm{b}}$, Brant C. Faircloth ${ }^{\mathrm{c}}$, Axel Meyer $^{\mathrm{a}}$, Michael E. Alfaro ${ }^{\mathrm{b}}$ \\ ${ }^{a}$ Department of Biology, University of Konstanz, Konstanz, Germany \\ ${ }^{\mathrm{b}}$ Department of Ecology and Evolutionary Biology, University of California, Los Angeles, CA, USA \\ ${ }^{\mathrm{c}}$ Department of Biological Sciences and Museum of Natural Science, Louisiana State University, Baton Rouge, LA, USA
}

Keywords:

Adaptive radiation

East African rift lakes

Niche constraint

Phylogeny

\begin{abstract}
A B S T R A C T
Adaptive radiations could often occur in discrete stages. For instance, the species flock of $\sim 1000$ species of Lake Malawi cichlid fishes might have only diverged once between rocky and sandy environments during the initial stage of their diversification. All further diversification within the rock-dwelling (mbuna) or sand-dwelling (utaka) cichlids would have occurred during a subsequent second stage of extensive trophic evolution that was followed by a third stage of sexual trait divergence. We provide an improved phylogenetic framework for Malawi cichlids to test this three-stage hypothesis based on newly reconstructed phylogenetic relationships among 32 taxonomically disparate Malawi cichlids species. Using several reconstruction methods and 1037 ultra-conserved element (UCE) markers, we recovered a molecular phylogeny that confidently resolved relationships among most of the Malawi lineages sampled when a bifurcating framework was enforced. These bifurcating reconstructions also indicated that the sanddwelling species Cyathochromis obliquidens was well-nested within the primarily rock-dwelling radiation known as the mbuna. In contrast to predictions from the three-stage model of vertebrate diversification, the recovered phylogeny reveals an initial colonization of rocky reefs, followed by substantial diversification of rock-dwelling lineages, and then at least one instance of subsequent evolution back into sandy habitats. This repeated evolution into major habitat types provides further evidence that the three-stage model of Malawi cichlid diversification has numerous exceptions.
\end{abstract}

\section{Introduction}

The incredibly species rich radiation of cichlid fishes in Lake Malawi ( $\sim 1000$ species) has been hypothesized to provide a model of ecological divergence that many radiations mirror: evolution along three predictable niche axes in temporally discrete stages (Danley and Kocher, 2001; Streelman and Danley, 2003). This three-stage model posits that adaptively radiating clades diverge predictably and sequentially: first along a habitat axis, then along a trophic axis, and finally along a signaling, or sexual trait, axis. The three-stage model has strongly influenced the study of adaptive radiation and has been used to characterize diversity in cichlid lineages ranging from Central America to the East African rift lakes (Salzburger, 2009; Martin and Genner, 2009; Hulsey et al., 2010; Parnell and Streelman, 2011; Kautt et al., 2012; López-Fernández et al., 2012; Hulsey et al., 2013a; Husemann et al., 2014;

\footnotetext{
* Corresponding author at: Department of Biology, University of Konstanz, Universitätstraße 10, Konstanz 78457, Germany.

E-mail address: darrin.hulsey@uni-konstanz.de (C.D. Hulsey).
}

Muschick et al., 2014; Salzburger et al., 2014; Santos-Santos et al., 2015; Ivory et al., 2016; Malinsky and Salzburger, 2016). This hypothesis of niche evolution has also been invoked as a putative explanation for diversification in a large number of other disparate clades including plants, invertebrates, and other vertebrate groups (Ackerly et al., 2006; Cowman et al., 2009; Harmon et al., 2008; Gavrilets and Losos, 2009; Arnegard et al., 2010; Glor, 2010; Sallan and Friedman, 2012). However, despite its widespread usage as a model for adaptive radiation, no explicit phylogenetic test of the three-stage model has been made in the group where it was first formulated, the Lake Malawi cichlid fishes.

In both freshwater and marine environments, transitions onto reefs could be key to determining how a clade diversifies (Alfaro et al., 2007; Price et al., 2011; Hodge et al., 2012; Price et al., 2013; Tornabene et al., 2015), but specialization to this habitat could also represent an evolutionary dead end (Alroy, 2008; Kiessling and Simpson, 2011). Reef-dwelling lineages might only rarely or never produce species that evolve to colonize other habitats. For instance, the mbuna constitute a putatively monophyletic group of approximately 400 cichlid species that could only inhabit 
the rocky shores of Lake Malawi (Ribbink et al., 1983; Genner et al., 2004; Genner and Turner, 2005). The habitat complexity that characterizes the rocky shores of Lake Malawi provides rich opportunities for niche partitioning and trophic specialization, and the mbuna do dominate and extensively exploit this habitat (MacArthur and Levins, 1964; Schoener, 1974; Ribbink et al., 1983; but see Martin and Genner, 2009). But, these rocky reefs could also have provided a habitat niche that was impossible for the mbuna to escape once they colonized it. For instance, modifications for algivory that is the dominant mode of mbuna feeding or the evolution of specific locomotory abilities associated with navigating complex rocky environments might have led to ecological specialization that was extremely difficult to reverse (Schluter, 2000; Alfaro et al., 2007; Price et al., 2011; Rupp and Hulsey, 2014). Once they initially colonized the rocky reefs in Malawi, the mbuna clade may also have entered a stage in which sexual selection and trophic evolution exclusively drove their diversification (Streelman and Danley, 2003; Malinsky and Salzburger, 2016). Phylogenetic analyses could help to resolve whether major habitat shifts back to sandy habitats have occurred during the mbuna radiation.

When traditional phylogenetic approaches have been used to reconstruct relationships among Malawi cichlids, they have often produced trees with limited resolution. Recovering robust phylogenetic hypotheses for Malawi cichlids has proven to be challenging due both to the young age of the entire clade ( $\sim 2$ million years) and the high potential for hybridization within this largely sympatric radiation (Kocher et al., 1995; Albertson et al., 1999; Hulsey et al., 2010; Mims et al., 2010; Brawand et al., 2014; Genner et al., 2015). Most previous inferences of Malawi cichlid phylogeny have been based primarily on mitochondrial DNA sequences (Kocher et al., 1995; Meyer et al., 1996; Moran and Kornfield, 1993; Hulsey et al., 2007, 2010; York et al., 2015). However, the rampant shared polymorphism and limited resolution provided by physically linked mitochondrial markers provides little confidence in the phylogenetic position of most lineages within the Malawi radiation (Moran and Kornfield, 1993; Meyer, 1994; Won et al., 2005; Hulsey et al., 2013b; Brawand et al., 2014). Advantageously, next-generation sequencing technologies offer enormous promise for resolving even the most intractable of phylogenetic problems. For instance, sequence capture of regions anchored by ultra-conserved elements (UCEs) offers an efficient means of generating massive genomic data sets capable of resolving phylogenetic relationships at both deep and shallow scales (Bejerano et al., 2004; Faircloth et al., 2014). UCEs have become increasingly popular as phylogenetic markers and have been used to reconstruct evolutionary trees for ancient clades as divergent as mammals, fishes, birds, turtles, and arthropods (Crawford et al., 2012; Faircloth et al., 2014; 2013; McCormack et al., 2012). However, one of the most compelling characteristics of UCEs for use in systematics is that the flanking regions increase in variable sites, and thereby phylogenetically informative changes, as the distance from the UCE center increases (Faircloth et al., 2012). This variation, in theory, should allow for better resolution of nodes across a range of evolutionary timescales potentially even including the short timeframe over which Malawi cichlids have diversified (McGee et al., 2016).

If a robust phylogeny of Malawi cichlids were reconstructed, there are several lineages that could violate the pattern of diversification predicted from a strict interpretation of the three stage model (Konings, 1991). Within what are generally considered utaka, or non-mbuna, there are a number of species that have likely invaded rocky reefs. The algivorous species Protomelas taeniolatus is trophically similar to many mbuna but is likely more closely related to groups that are largely sand-dwelling (Ribbink et al., 1983). Other non-mbuna species such as Cheilochromis euchi- lus that possess hypertrophied lips are also likely specialized to feed on invertebrates located in the rocky crevices of these reef habitats (Konings, 1991; Baumgarten et al., 2015; Henning et al., 2017). A number of piscivores such as Tyrannochromis nigriventer also commonly feed on fishes that exploit rocky reefs (Ribbink et al., 1983). Additionally, there are several lineages of mbuna that exploit non-rocky habitats and currently have unclear phylogenetic affinities. For instance, the species Maylandia livingstonii lives and breeds primarily in sandy habitats and putatively belongs to a genus that is otherwise largely confined to rocky reefs (Fryer and Iles, 1972; Ribbink et al., 1983; Konings, 1991). Likewise, the currently ambiguous phylogenetic position of the sand-dwelling species Cyathochromis obliquidens has consequences for our understanding of both habitat and trophic diversification in Malawi cichlids (Fig. 1). C. obliquidens has frequently been considered to be closely allied to the mbuna clade (Fryer and Iles, 1972; Ribbink et al., 1983; Hulsey et al., 2010). Yet, C. obliquidens appears to both scrape aufwuchs that coat leaves as well as take bites from plants in the genus Vallisneria (Fryer, 1959; Ribbink et al., 1983). These plants grow almost exclusively in Lake Malawi's sandy habitats (Konings, 1991). If C. obliquidens were the sister group to the other mbuna, the ubiquitous habit of scraping algae in the mbuna (Fryer and Iles, 1972; Ribbink et al., 1983; Rupp and Hulsey, 2014) might have evolved from herbivory on vascular plants. Alternatively, trophic specialization in C. obliquidens could have involved a transition from the mbuna habit of scraping algae to sometimes eating vascular plant material. Also, if $C$. obliquidens were well nested within the mbuna radiation, this placement would suggest that following extensive diversification on the rocky outcrops of Lake Malawi, a member of the mbuna clade diverged to exploit sandy habitats. Alternatively, if $C$. obliquidens were more closely related to non-mbuna living in sandy habitats or was found to be the sister lineage to the 400 species of rock-dwelling mbuna, then it would still be possible that the radiation of mbuna once it evolved to exploit reefs might have remained confined to these rocky habitats (Streelman and Danley, 2003; Malinsky and Salzburger, 2016). Robust phylogenies of Malawi cichlids could be used to test for this extreme niche conservatism and to evaluate through a more complete understanding of Malawi cichlid relationships the strictest interpretation of the three-stage model.

To provide a comparative framework for Malawi cichlid evolution and to test a particular case of whether adaptive divergence in Lake Malawi cichlids is consistent with the three-stage model, we generated several nuclear sequence based phylogenetic hypotheses. First, sequences of 1037 UCE loci were generated for many representatives of previously delineated major lineages within Lake Malawi. Then, using several methods for tree searching, the robustness of relationships to different reconstruction methods was determined. Finally, we assessed the phylogenetic affinities of the sand-dwelling species $C$. obliquidens to evaluate its placement with respect to the larger rock-dwelling mbuna radiation to determine whether the patterns of habitat and trophic evolution in Malawi cichlids are strictly consistent with the three stage model.

\section{Materials and methods}

\subsection{Taxa sampled}

Phylogenetic hypotheses were reconstructed using a set of UCEs that have been sequenced previously (McGee et al., 2016) and are here combined with sequences of 16 new species of cichlids from Lake Malawi (Table 1). In total, nine named genera in the mbuna were included along with representatives of 17 genera of Lake Malawi cichlids that are not likely nested within the mbuna clade. Additionally, data from the species Pundamillia nyeri, Haplochromis 
A

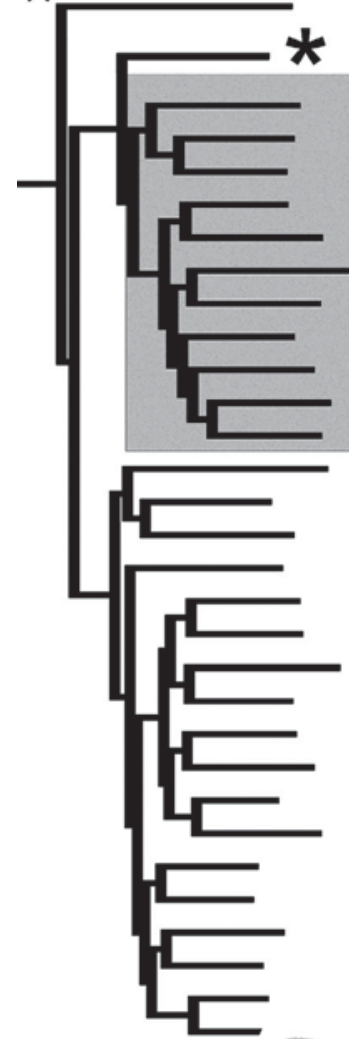

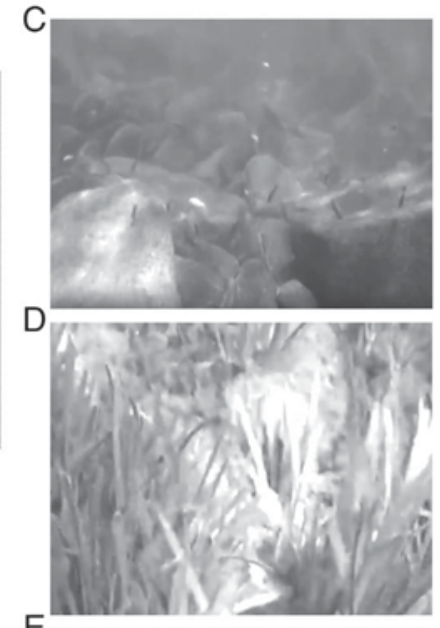

$\mathrm{E}$
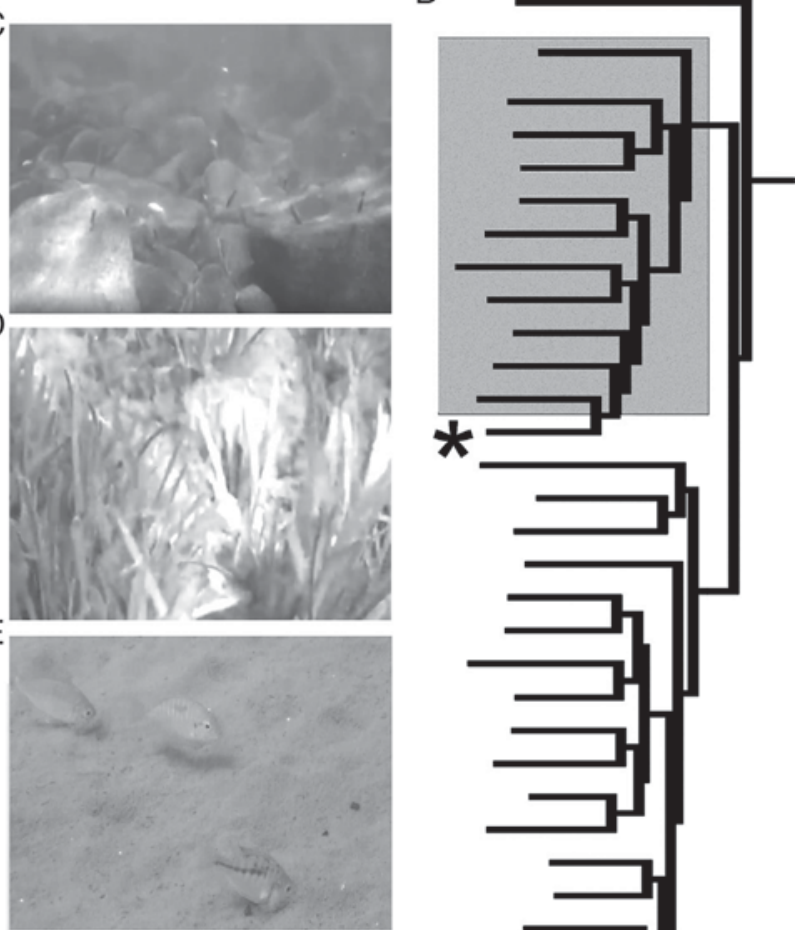

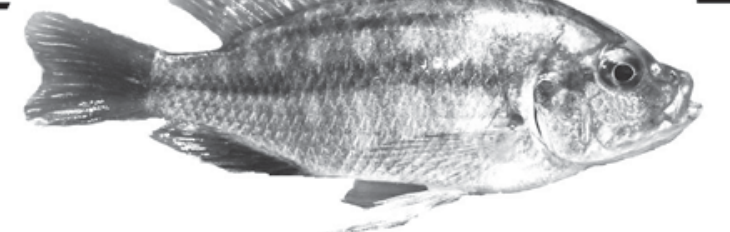

Cyathochromis obliquidens

Fig. 1. Two possible alternative (A, B) phylogenetic positions for Cyathochromis obliquidens highlighted with a large asterisk $\left({ }^{*}\right)$. If this species is phylogenetically located outside of the mbuna clade (gray) or even sister to it (A), it would suggest the mbuna have effectively only evolved to exploit rocky reefs (C). This would be consistent with the mbuna showing no further major habitat transitions during evolution within Lake Malawi and would be most consistent with the strict interpretation of the three-stage model. Alternatively (B), if C. obliquidens is phylogenetically nested within the mbuna it would indicate this clade has simultaneously evolved the ability to feed on Vallisneria (D) and to re-exploit sandy habitats (E), the other major substrate type in Lake Malawi. This would be inconsistent with the strictest interpretation of the three-stage model.

burtoni, Simochromis babaulti, Neolamprologus brichardi, Bathybates minor, and Oreochromis niloticus were included to phylogenetically polarize our Malawi reconstructions (Meyer et al., 1996; Friedman et al., 2013; Brawand et al., 2014; McGee et al., 2016). For newly sequenced individuals, fin and/or muscle tissue were taken from each specimen, library preparation performed, and UCE sequencing analyses completed as has been previously described (Faircloth et al., 2012, 2013) but that is also detailed below.

\subsection{DNA extraction and library preparation}

DNA was extracted from 5 to $15 \mathrm{mg}$ of ethanol-preserved tissues. These extractions followed a modified version of the Qiagen DNEasy protocol, which utilizes $65 \mathrm{uL}$ of warm $\left(50-55^{\circ} \mathrm{C}\right)$ buffer $\mathrm{AE}$ instead of the recommended $200 \mathrm{uL}$ at room temperature. Following elution, we quantified extraction efficiency using a Qubit 2.0 Fluorometer by thoroughly mixing $2.0 \mathrm{uL}$ eluate with $198 \mathrm{uL}$ of fluorescent dye solution. To ensure high-quality extracts, 50-100 ng of each extract were visualized via electrophoresis using a $1.5 \%$ agarose gel in TBE. $100 \mathrm{uL}$ aliquots were then prepared for each specimen that had been equilibrated to a DNA concentration of $10 \mathrm{ng} / \mathrm{uL}$ and then were sonicated using a BioRuptor
(Diagenode, Inc.). Each sample was sheared to generate products of $300-500 \mathrm{bp}$ in length that were then size validated with gel visualizations.

Following sonication, libraries were prepared according to a modified version of the Illumina library preparation protocols from Faircloth et al. (2014). In preparing pooled DNA libraries, we used a series of standard library preparation reagents (Kapa Biosystems, Inc.) combined with dual-indexing adaptors (Glenn et al., 2016) that were added during the PCR amplification phase. This substantially reduced the number of primer tags needed to uniquely identify and differentiate libraries. Nucleic acid concentrations of pre-amplification libraries were immediately quantified. Following this quantification, we prepared a $50 \mathrm{uL}$ PCR reaction mix consisting of 15-20 uL DNA library, $25 \mathrm{uL}$ HiFi HotStart ReadyMix polymerase, $5 \mathrm{uL}$ primer mix, and 0-5 uL double-distilled water (ddH2O). The following thermal cycle configuration was used: $98^{\circ} \mathrm{C}$ for $45 \mathrm{~s}, 10-16$ cycles of $98^{\circ} \mathrm{C}$ for $15 \mathrm{~s}, 60^{\circ} \mathrm{C}$ for $30 \mathrm{~s}, 72^{\circ} \mathrm{C}$ for $60 \mathrm{~s}$, then $72{ }^{\circ} \mathrm{C}$ for an extended $5 \mathrm{~min}$, and an indefinite hold at $4{ }^{\circ} \mathrm{C}$. As a final step, the resulting reactions were purified with 1.8X Serapure solution (Rohland and Reich, 2012; Glenn et al., 2016), two 80\% EtOH washes, and then rehydrated with $23 \mathrm{uL}$ 10 -mM Tris buffer. 
Table 1

The new species sequenced for this study and collected in 2010, the collection location in Lake Malawi, the number of UCE loci sequences used in the analyses for each species, and their Genbank accession numbers.

\begin{tabular}{|c|c|c|c|}
\hline Species & $\begin{array}{l}\text { Collection } \\
\text { Locality }\end{array}$ & $\begin{array}{l}\text { Number of } \\
\text { Loci }\end{array}$ & $\begin{array}{l}\text { Genbank } \\
\text { Accessions }\end{array}$ \\
\hline $\begin{array}{l}\text { Copadichromis } \\
\text { trimaculatus }\end{array}$ & Chinyamwezi & 1026 & KARH00000000 \\
\hline Ctenopharynx pictus & Mumbo & 1026 & КАТВ00000000 \\
\hline Cyathochromis obliquidens & Otter Point & 1023 & KARJ00000000 \\
\hline Docimodus evelynae & Boadzulu & 1015 & KARM00000000 \\
\hline Genyochromis mento & Thumbi West & 1010 & KARO00000000 \\
\hline Hemitilapia oxyrhynchus & Otter Point & 1018 & KARP00000000 \\
\hline Labidochromis gigas & Thumbi West & 1025 & KART00000000 \\
\hline Mchenga conophoros & Mazinizi Reef & 1015 & KARC00000000 \\
\hline Metriaclima greshakae & $\begin{array}{l}\text { Makakola } \\
\text { Reef }\end{array}$ & 1020 & KASK00000000 \\
\hline Metriaclima patricki & Mbenji & 1016 & KASP00000000 \\
\hline Otopharynx heterodon & Thumbi West & 1013 & KASZ00000000 \\
\hline Petrotilapia nigra & Thumbi West & 1020 & KATI00000000 \\
\hline Stigmatochromis woodi & Choifu & 1025 & KATU00000000 \\
\hline $\begin{array}{l}\text { Taeniolethrinops } \\
\text { praeorbitalis }\end{array}$ & Otter Point & 1022 & КАТХ00000000 \\
\hline Tropheops microstoma & Otter Point & 1021 & KAUD00000000 \\
\hline $\begin{array}{l}\text { Tyrannochromis } \\
\text { nigriventer }\end{array}$ & Thumbi West & 1022 & KAUG00000000 \\
\hline
\end{tabular}

\subsection{Library enrichment and sequencing}

To prepare each library for enrichment, they were combined into pools of equimolar ratios ( $\sim 500 \mathrm{ng}$ per pool). To normalize the volumes of each pool, we dried down them in a SpeedVac and rehydrated each in $3.4 \mathrm{uL}$ Tris buffer. Based on the sequence capture protocol available at ultraconserved.org, libraries were enriched for UCE targets using the following reagents: (1) $100 \mathrm{ng}$ of the MYBaits UCE Capture Kit baits (MYcroarray, Inc.) (2) 500 ng blocking oligos designed against our custom dual sequence indexes, (3) MYcroarray MySelect hybridization solutions (MYcroarray, Inc.), and (4) 1\% SDS (versus 10\% SDS). The hybridization reaction was run for $24 \mathrm{~h}$ at $65{ }^{\circ} \mathrm{C}$, allowing the capture probes to bind to UCE targets. Upon completion, we thoroughly mixed streptavidin-coated beads (MyOne C1, Life Technologies, Inc.) with the hybridized pools and then washed the bound libraries according to the protocol. Beads were then rehydrated in $33 \mathrm{uL}$ of ddH2O, amplified with $15 \mathrm{uL}$ of the mix in a post-hybridization limited cycle PCR recovery step, and the end products quantified using a Qubit fluorometer (Faircloth et al., 2014). After qPCRquantification of the enriched, double-indexed pools using a library quantification kit (Kapa Biosystems), we created an equimolar solution of all pools at a total concentration of $10 \mathrm{nM}$. These libraries were then shipped to the Georgia Genomics Facility and sequenced using the Illumina NextSeq PE150 platform.

\subsection{Sequence data assembly and alignment}

After sequencing, we trimmed adapters, low quality bases, and sequences containing ambiguous base calls using the Illumiprocessor tool (https://github.com/faircloth-lab/illumiprocessor) that is a wrapper for the trimmomatic package (Bolger et al., 2014). The reads were assembled on a species-by-species basis into contigs using Trinity v2013-02-25 (Grabherr et al., 2011). Following assembly, the PHYLUCE software package (Faircloth, 2016) containing a custom Python program that integrates LASTZ to align species-specific contigs to the set of UCE probes was used for enrichment (Faircloth et al., 2013; McGee et al., 2016). This program simultaneously removes reciprocal and non-reciprocal duplicate hits from the data set. During matching, this program creates a relational database of matches to UCE loci by taxon. After generat- ing the relational database of matches to enriched sequences and genome-enabled taxa, we used additional components of PHYLUCE to query the database and generate fasta files for the UCE loci we identified across all taxa (Faircloth, 2016). Following enrichment and sequencing, contigs that matched no UCEs and contigs that also matched multiple loci were removed. Using the remaining set of contigs, a matrix was generated that included only UCE loci that were recovered from $95 \%$ of the species examined. Then, we used a custom Python program to align contigs with MAFFT and trim contigs representing UCEs, in parallel, across the selected taxa prior to phylogenetic analysis (Katoh et al., 2005; Faircloth et al., 2012). The data are available on Genbank's SRA database (Table 1).

\subsection{Phylogenetic reconstruction}

To estimate sequence divergence and reconstruct phylogenetic hypotheses from our data, we concatenated our UCE alignments into a PHYLIP-formatted super-matrix (Felsenstein, 2005). To summarize the amount of genetic differentiation between several putative monophyletic groups, the percent uncorrected-pairwise sequence divergence was estimated based on these alignments. Partition schemes for a 95\% complete data matrix were then assigned using the relaxed clustering algorithm implemented in PartitionFinder v2.0.0 (Lanfear et al., 2012).

To reconstruct phylogenetic trees for the UCE loci using the program SNAPP (Bouckaert et al., 2014), we utilized a de novo SNP calling approach by aligning all raw reads against the sample with the highest coverage across all UCE loci. This method integrates BWA v. 0.7.7-1 and PICARD v. 1.106 (http://picard.sourceforge.net/) to output alignments in BAM format, repairs any formatting violations, adds read group header information, and marks duplicates in each BAM. We then merged all resulting BAMs into one file, realigned the data and called SNPs and indels using GATK v. 3.5. To ensure high-quality SNPs in downstream analyses, the data was hierarchically filtered according to stringent quality and validation parameters, excluding SNPs with QUAL scores under 25, low variant confidence, and poor validation. Finally, the resulting data was filtered further using VCFTOOLS v. 0.1.14 (Danecek et al., 2011) to remove all loci that missed SNP calls for over $25 \%$ of the species. We converted the filtered file to SNAPP format (Bryant et al., 2012) using a program from PHYLUCE (Faircloth, 2016). The SNP data was then uploaded to the SNAPP module that was run in BEAST v.2.2.1 (Bouckaert et al., 2014). The analysis ran for $5,000,000$ generations, and convergence was assessed for stable posterior likelihood and ESS values of over 200.

We also carried out phylogenetic reconstruction on a $95 \%$ complete matrix with a GTR + gamma partitioning scheme on all variable sites using RAxML 8.0.19 (Stamatakis, 2014) and the PTHREADS binary. Initially, 20 maximum-likelihood (ML) searches were conducted to find the best-fitting phylogenetic hypothesis. Then, we generated non-parametric bootstrap replicates under the autoMRE flag which runs the analysis until convergence. Upon completion, the best fit ML tree was reconciled with the bootstrap replicates to generate node support values.

For our third method of reconstruction, we used the MPI version of ExaBayes v1.4.1 (Aberer et al., 2014) employing 12 threads to conduct four independent runs with Metropolis-Coupling to expedite convergence. One heated chain was used to sample the posterior distribution more efficiently and to avoid getting stuck in local optima, which did appear to occur based on visual inspection of preliminary trace files using Tracer (Rambaut et al., 2014). We assessed convergence based on our examination of the traces, associated ESS values, as well as the standard deviation of the split frequencies generated from ExaBayes. Using the consense program, a consensus tree was created from the four independent runs 
(Felsenstein, 2005). Tree files from the three reconstruction methods are available as Supplementary materials.

Because of the recent history and potential for hybridization among Malawi cichlids, our data might generally provide little support for a phylogenetic scenario with largely bifurcating structure as the methods above enforce. The history of Malawi cichlids might be more consistent with extensive reticulation and/or even large nuclear data sets might have insufficient power to infer a bifurcating structure. Also, individual lineages might show particularly high or low evidence for reticulation in the network. To explore these possibilities, we implemented the distance based method neighbor-net (Bryant and Mouton, 2004) in the program SplitsTree4 (Huson and Bryant, 2006). The neighbor-net approach generates a collection of weighted splits using a neighbor-joining distance algorithm that can return either bifurcating or reticulate networks among species. If the data were generally bifurcating, the network produced should be largely tree-like. Alternatively, a network with extensive reticulation would be found when relationships among taxa in the neighbor-net were generally unresolved or formed box-like edges. Poorly resolved phylogenetic trees recovered using SNAPP, RaxML, and ExaBayes coupled with non-bifurcating phylogenetic networks would together suggest that future analyses incorporating both more data and different reconstruction methods will likely be necessary to more fully understand the evolutionary relationships in the Lake Malawi radiation.

\section{Results and discussion}

Our phylogenomic analyses provided resolution on many relationships among the Malawi cichlids. Additionally, the isolation and phylogenetic reconstruction of UCEs provided the resolution to evaluate whether patterns of bifurcating divergence support the three stage model. Following enrichment and sequencing, an average of $2,254,432$ reads were obtained per species. The analyzed matrix including only loci that were recovered from $95 \%$ of the species examined, and this constituted 1037 UCEs that had an average length of $359 \mathrm{bp}$. The assembled character matrix included 553,632 sites and the dataset contained a total of 13,698 informative SNPs.

As has been found previously in studies of Malawi cichlid molecular evolution (Loh et al., 2008; Hulsey et al., 2010; Friedman et al., 2013; Brawand et al., 2014), there was much less than $1.0 \%$ pairwise nuclear sequence divergence among the entire flock (Table 2). The maximum nuclear sequence divergence estimated for all of the UCE loci across the Malawi flock was $0.183 \%$ and this was between the mbuna Genyochromis mento and Rhamphochromis longiceps. The mbuna showed a maximum of $0.137 \%$ divergence and the non-mbuna sand dwelling species (when $R$. longiceps was removed) showed a maximum sequence divergence of $0.140 \%$. There were three monophyletic congeneric species pairs recovered in all analyses and they showed highly

Table 2

Uncorrected percent sequence divergence for the 1037 UCE loci alignments. The mean, minimum, and maximum percent sequence divergence in several monophyletic genera, the mbuna, the utaka, and Rhamphochromis versus the remainder of the Malawi cichlids are given. The sequence divergence between the two species sampled in the genera Chilotilapia, Labeotropheus, Melanochromis, and Metriaclima were summarized as "Monophyletic Genera".

\begin{tabular}{llll}
\hline Clades & Mean & Minimum & Maximum \\
\hline Monophyletic Genera & 0.089 & 0.084 & 0.094 \\
mbuna & 0.107 & 0.087 & 0.137 \\
utaka & 0.106 & 0.059 & 0.140 \\
Rhamphochromis vs. Malawi & 0.163 & 0.149 & 0.183 \\
\hline
\end{tabular}

similar levels of percent sequence divergence: Labeotropheus were $0.087 \%$ divergent, Melanochromis were $0.089 \%$ divergent, and Cheilotilapia were $0.084 \%$ divergent. Although the UCE loci should be relatively conserved compared to much of the genome (Bejerano et al., 2004; Faircloth et al., 2014), this level of sequence divergence suggests Malawi congeneric species might commonly have nuclear genomes that are differentiated at only one base pair for every thousand nucleotides.

Our strictly bifurcating reconstructions provided the most robust phylogenetic framework of Malawi cichlid evolutionary relationships produced to date (Fig. 2). There was $100 \%$ posterior support for Rhamphochromis longiceps as sister to the remaining diversity of the Malawi cichlids examined under RaxML and ExaBayes although SNAPP placed this species as sister to the other sand-dwelling species with equally strong support. Rhamphochromis has long been considered as part of a relatively distinct evolutionary lineage in the Malawi radiation (Meyer, 1993; Meyer et al., 1996; Shaw et al., 2000; Hulsey et al., 2007, 2013; McGee et al., 2016). All three bifurcating reconstruction methods recovered unambiguous monophyly of the other major lineages composing the largest radiation of sand-dwelling cichlids. Aulonocara stuartgrantii was recovered as the sister group to this large clade of Malawi cichlids that do not inhabit rock environments. In most mitochondrial phylogenies published to date, Aulonocara has been inferred to be more closely related to the mbuna (Hulsey et al., 2010). Likewise, the fatlip cichlid Placidochromis milomo that has been inferred to be part of the mbuna radiation based largely on mitochondrial sequences of ND2 (Hulsey et al., 2007) is nested well outside the mbuna radiation, and it is more closely related to other non-mbuna species like the similarly fat-lipped Chilotilapia euchilus. Some other notable affinities that we recovered in these reconstructions were the grouping of the piscivores Aristochromis chrysti and Tyrannochromis nigriventer. The monophyly of these two species as well the two exemplars of the genus Labeotropheus, Melanochromis, and Chilotilapia were recovered using all three bifurcating reconstruction methods. This degree of resolution and support within the radiation suggests that a phylogenomic focus that uses the sequencing of UCE loci could provide substantial power for differentiating the relationships among Malawi cichlids even at very close taxonomic scales.

The monophyly of the rock-dwelling mbuna species + Cyathochromis obliquidens was also clearly supported in all three bifurcating reconstructions (Fig. 2). The two Labeotropheus species that possess the unique condition of having rectangular-shaped mouths (Fryer and Iles, 1972; Konings, 1991) were also consistently recovered as the sister group to the remaining mbuna taxa. This result is concordant with some other phylogenetic analyses that have utilized a relatively small number of nuclear SNPs (Hulsey et al., 2013a). The fin-biter Genyochromis mento and the two species of Melanochromis (Konings, 1991) both have a relatively elongated body shape and were recovered as closely related. The two species Metriaclima greshakae and $M$. patricki, were also recovered as monophyletic in the RAxML and ExaBayes inferred phylogenies.

In the most well resolved bifurcating topology found using ExaBayes, there were a number of relationships that were highly supported (80-100\% posterior probabilities) that were not recovered from the other two bifurcating reconstruction methods (Fig. 2). Aulonocara stuartgranti, Mchenga conophoros and Taeniolethrinops praeorbitalis grouped with one another. Otopharynx heterodon and Hemitilapia oxyrhynchus were also recovered as close relatives. Fossochromis rostratus and Nimbochromis polystigma were found to be members of a clade containing the two highly piscivorous species Aristochromis chrystyi and Tyrannochromis nigriventer. Additionally, Placidochromis electra and the fatlip species $P$. milomo were recovered as close relatives of Chilotilapia rhoadesii and another fatlip species $C$. euchilus. The ExaBayes analyses also suggested the two 
A

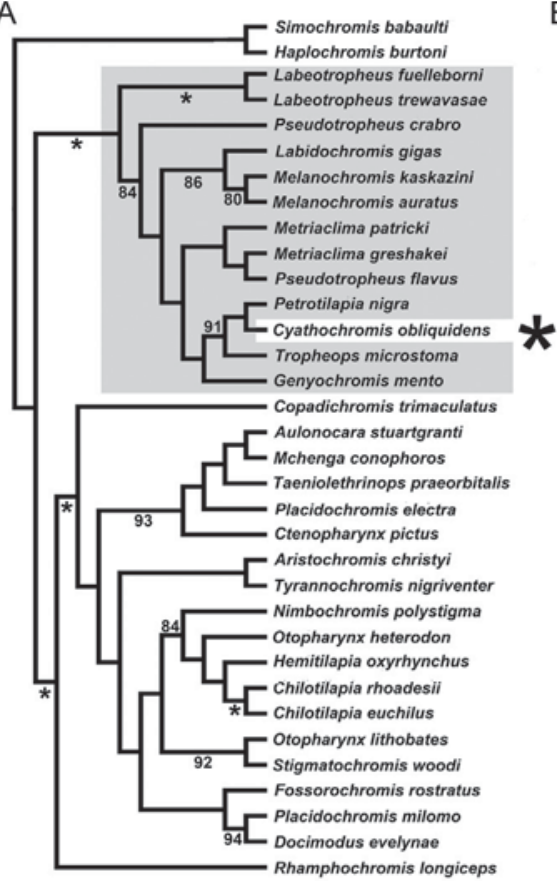

B

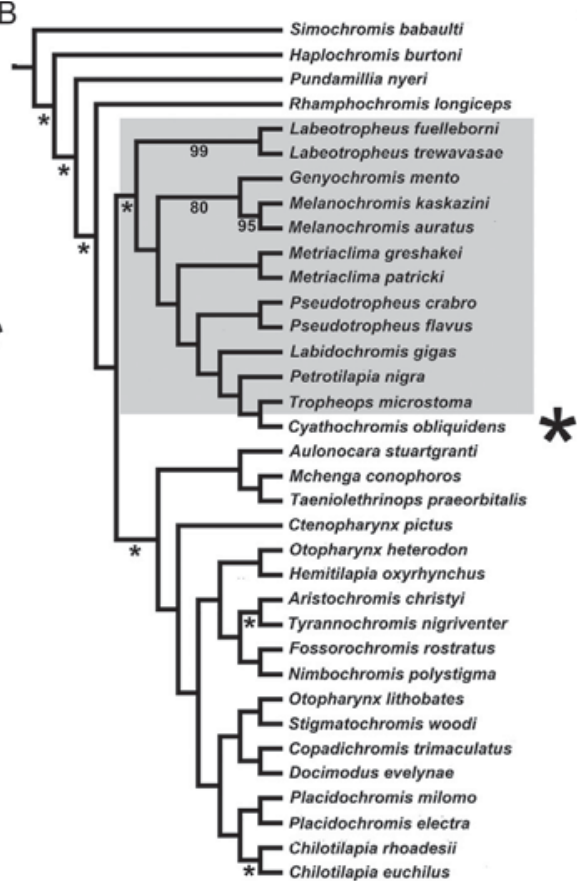

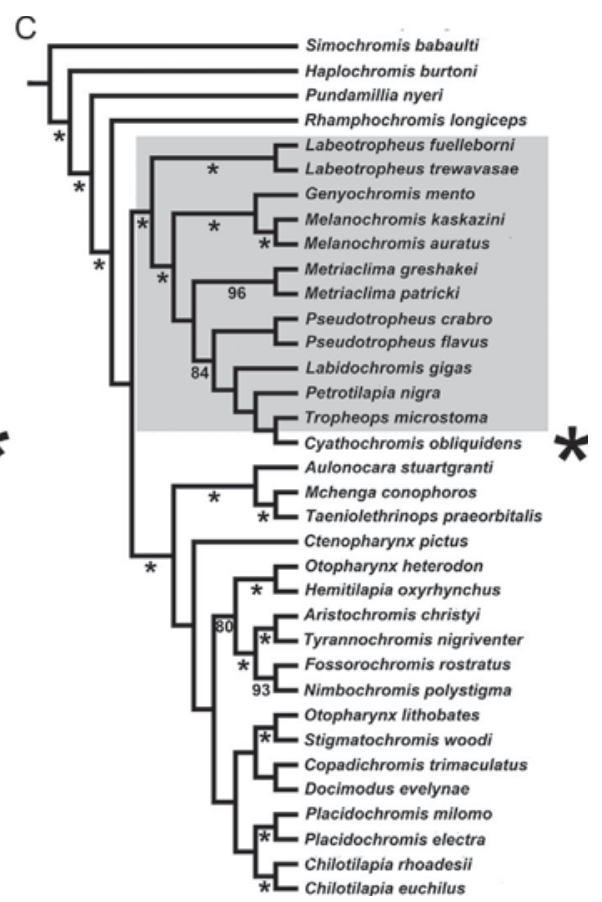

Fig. 2. Phylogenetic reconstructions of Lake Malawi cichlids. The posterior probabilities from the concatenated Bayesian analysis greater than $50 \%$ are depicted behind nodes. If there was $100 \%$ posterior probability support for a node, it is indicated with a small asterisk. The clade that includes the members of the rock-dwelling Mbuna clade is depicted in gray. The inferred phylogenetic position of $C$. obliquidens, that is consistently found to be nested within the mbuna, is demarcated with a large asterisk in the reconstructions from SNAPP (A), RAxML (B), and ExaBayes (C).

Pseudotropheus species examined had close affinities to Labidochromis gigas, Petrotilapia nigra, Tropheops microstoma, and Cyathochromis obliquidens.

Forcing the Malawi radiation into a strictly bifurcating phylogenetic history may be problematic because of the age of the radiation and potential for interspecific gene flow (Kocher et al., 1995; Albertson et al., 1999; Hulsey et al., 2010; Mims et al., 2010; Brawand et al., 2014; Genner et al., 2015). Our distance based phylogenetic network is consistent with extensive gene flow and nonbifurcating relationships among most of the lineages in Malawi (Bryant and Mouton, 2004; Fig. 3). Even with the 1000 loci, there appear to only be three relatively distinct major clusters in this network: Rhamphochromis longiceps, the non-mbuna, and mbuna. Relationships were generally unresolved within these clusters and not strictly bifurcating (Fig. 3). These inferences for reticulate evolution could also explain why several of the relationships using bifurcating tree reconstructions had little resolution (Fig. 2). Future analyses will likely have to either increase the number of loci sampled, utilize long sequence reads that contain more informative changes per sequence, or employ different models of evolution such as species tree analyses to further our understanding of Malawi cichlid relationships. Determining the relative importance of strictly bifurcating lineage divergence, interspecific gene flow, and retained ancestral polymorphism will continue to challenge our ability to reconstruct the patterns of Malawi cichlid diversification.

Although the relationship of $C$. obliquidens was highly ambiguous with respect to all the other mbuna species in our neighbornet reconstruction, $C$. obliquidens was nested within the rockdwelling mbuna and found to have close affinities with species such as Petrotilapia nigra and Tropheops microstoma in all three bifurcating phylogeny reconstructions (Fig. 2). This result suggests that the trophic habit of biting Vallisneria leaves in $C$. obliquidens could have evolved from a more algivorous diet that characterizes many mbuna lineages. We did not perform a formal ancestral state reconstruction of this or the presence in rocky versus sandy habitats because our phylogeny only contains a small proportion of the several hundred extant species of rocky habitat dwelling mbuna. However, the inclusion of these species if they fall within the mbuna clade would likely only lend support to any inference of the paraphyly of the rocky dwelling habit for the mbuna clade. The clear nesting of $C$. obliquidens within the mbuna radiation strongly suggests that this species has, contrary to the threestage model, reinvaded the sandy habitats of Lake Malawi. Furthermore, it is likely that $C$. obliquidens simultaneously evolved the novel trophic habit of biting Vallisneria during its habitat reinvasion from rocky reefs into sandy habitats. Because this species might commonly eat algae off of Vallisneria leaves as well (Ribbink et al., 1983), these fish might only utilize the Vallisneria leaves themselves as a resource during times of extensive competition as expected under Liem's paradox (Robinson and Wilson, 1998). Regardless, this type of apparent coincident habitat and trophic divergence found in $C$. obliquidens is also not consistent with the idea that evolution along habitat and trophic niche axes has always happened discretely and independently during the Lake Malawi cichlid radiation.

Cyathochromis obliquidens is generally an exception to the diversification of the Lake Malawi mbuna that is otherwise largely constrained to rocky reefs (Ribbink et al., 1983; Reinthal, 1990). Yet, it is not the only deviation of Malawi cichlids from the three stage model (Konings, 1991; Hulsey et al., 2010). Within the nonmbuna, a number of species have invaded rocky reefs. The algivorous and mbuna-like species Protomelas taeniolatus is likely more closely related to groups that are largely sand-dwelling (Ribbink et al., 1983). Other non-mbuna species such as Cheilochromis euchilus with their hypertrophied lips have adapted to exploit Malawi's rocky reefs (Konings, 1991; Baumgarten et al., 2015; Henning et al., 2017; Fig. 2). Non-mbuna piscivores like Tyrannochromis nigriventer also commonly feed on fishes that exploit rocky reefs (Ribbink et al., 1983; Fig. 2). The relationships of these two species 


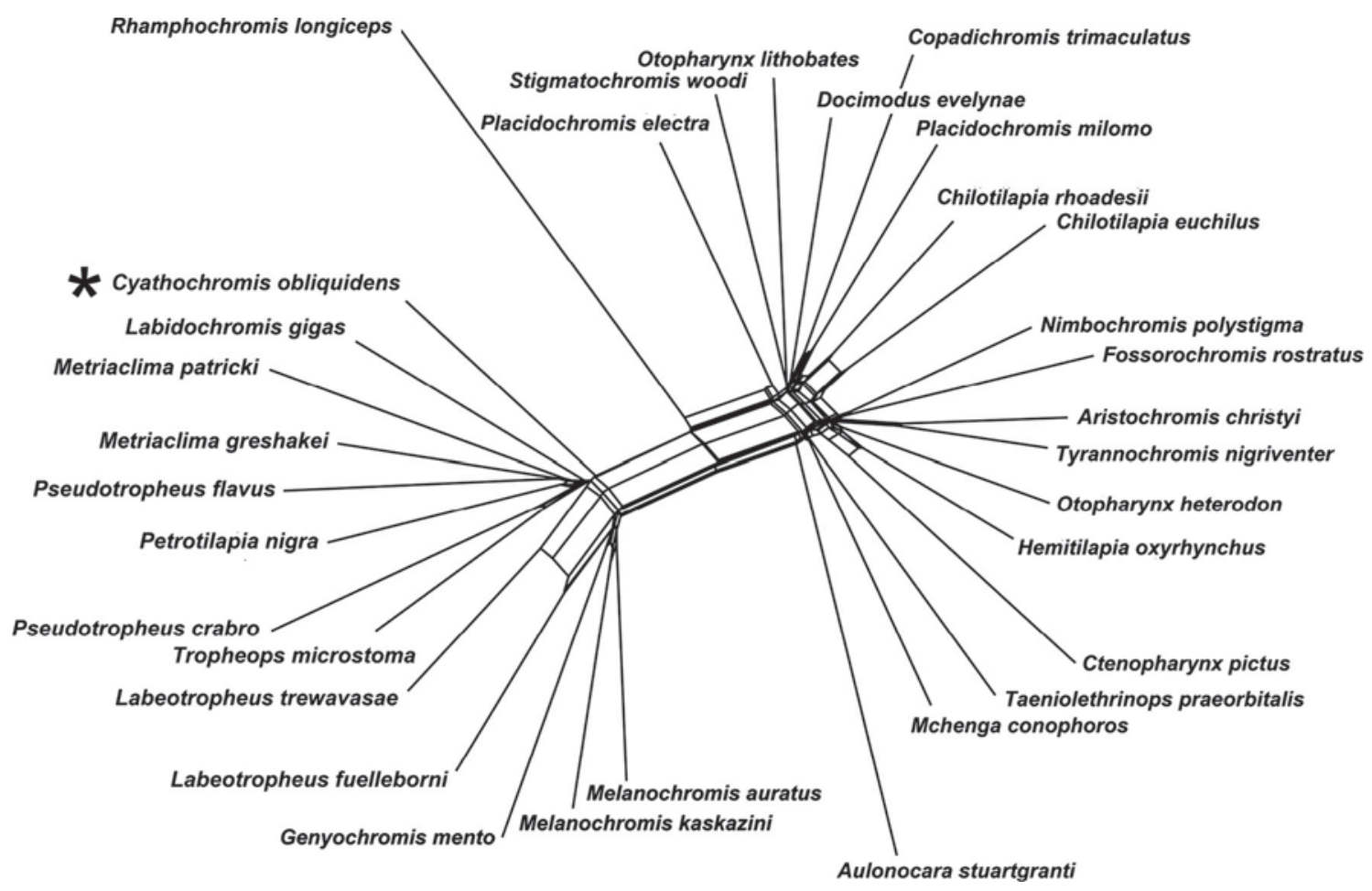

Fig. 3. To explore the potential for gene flow among branches, we generated a phylogenetic network of the Malawi cichlids sampled. Based on a neighbor-net reconstruction, extensive gene flow among most of the lineages in Malawi might be common. There appear to be three major clusters in this network: Rhamphochromis longiceps, the nonmbuna, and mbuna. Relationships are unresolved within these clusters and the relationships even among these three groups is inferred to be reticulate and not strictly bifurcating. Unlike in our strictly bifurcating reconstructions, the relationships of $C$. obliquidens are ambiguous with respect to all the other mbuna species in this network. Better models of evolution, more data, and detailed hypothesis testing of the tree-like versus reticulate structure of the radiation will likely all facilitate a better understanding of Malawi cichlid relationships.

in our bifurcating phylogenies are also not consistent with the three stage model. The mbuna species Maylandia livingstonii is another sand-dwelling species that is likely well nested within the mbuna (Fryer and Iles, 1972; Ribbink et al., 1983; Konings, 1991) and should be investigated in future phylogenetic tests of the three stage model. It seems clear that the divergence of Malawi cichlids into rocky and non-rocky habitats did not happen during only a single stage of this clade's radiation.

Additionally, other types of habitat related niche divergence within Malawi cichlids cast doubt on the primacy of this threestage model to explain cichlid diversification. Divergence along a benthic versus limnetic habitat transition has occurred multiple times within Lake Malawi (Hulsey et al., 2013a) as it has in many other aquatic systems (Schluter, 2000; Hollingsworth et al., 2013; Machado-Schiffiano et al., 2015; Kautt et al., 2016). Additionally, even within groups like the mbuna that are largely confined to rocky habitats, there is substantial divergence in species along depth gradients, what sizes of rocks are utilized for feeding, and even from what side of the rock algae is grazed (Ribbink et al., 1983; Parnell and Streelman, 2011; Rupp and Hulsey, 2014). The evolution of the mbuna into the rocky habitats of Lake Malawi did not halt their habitat niche diversification to a single stage of their adaptive radiation.

Phylogeny reconstruction that makes use of new genomic tools will continue to expand our understanding of the patterns of niche evolution in Lake Malawi and other adaptive radiations (Sidlauskas, 2008; Hulsey, 2009; Higham et al., 2015). Despite the extremely short timescales over which phylogenetic divergence has occurred in Malawi (Albertson et al., 1999; Won et al., 2005; Hulsey et al., 2010), as well as the rampant retention of ancestral polymorphism (Loh et al., 2008; Brawand et al., 2014), and the high potential for interspecific gene flow (Mims et al., 2010; Joyce et al., 2011), our analyses provide cautious optimism that we will be able to reconstruct a robust evolutionary framework for cichlid divergence in Lake Malawi. Because it is the most species rich radiation of fishes in the world (Fryer and Iles, 1972; Konings 1991; Danley and Kocher, 2001; Kocher 2004), this radiation will also continue to serve as a model for comparative biology. However, many evolutionary analyses of these fishes, including those focused on the three stage model, have either discounted the importance of phylogeny or relied on the limited inferences of relationships available from mitochondrial gene trees (Meyer et al., 1990; Meyer, 1993,1994; Danley and Kocher, 2001; Streelman and Danley, 2003; Hulsey et al., 2007; Fraser et al., 2009; York et al., 2015). Like any model that explicitly posits a particular sequence of evolutionary events, the three stage model depends on our understanding of evolutionary patterns of species divergence (Hollingsworth et al., 2013; Price et al., 2013). Markers like UCEs that allow a more comprehensive sampling of the nuclear genome will continue to facilitate the progression to a new stage of analyses in which evolutionary hypotheses, even in the most rapidly radiating lineages on earth, can be examined in a phylogenomic framework.

\section{Acknowledgements}

We thank the University of Malawi, Malawi Parks Service, and the Malawi government for logistics and permissions to collect fish in Malawi. This research was supported by a UCLA Faculty COR Grant to MEA. 


\section{References}

Ackerly, D.D., Schwilk, D.W., Webb, C.O., 2006. Niche evolution and adaptive radiation: Testing the order of trait divergence. Ecology 87, S50-S61.

Aberer, A.J., Kobert, K., Stamatakis, A., 2014. ExaBayes: massively parallel Bayesian tree inference for the whole-genome era. Mol. Biol. Evol. 31, 2553-2556.

Albertson, R.C., Markert, J.A., Danley, P.D., Kocher, T.D., 1999. Phylogeny of a rapidly evolving clade: the cichlid fishes of Lake Malawi, east Africa. Proc. Nat. Acad. Sci. U.S.A. 96, 5107-5110.

Alfaro, M.E., Santini, F., Brock, C.D., 2007. Do reefs drive diversification in marine teleosts? Evidence from the pufferfish and their allies (order Tetraodontiformes). Evolution 61, 2104-2126.

Alroy, J., 2008. Dynamics of origination and extinction in the marine fossil record. Proc. Nat. Acad. Sci. U.S.A. 105, 11536-11542.

Arnegard, M.E., McIntyre, P.B., Harmon, L.J., Zelditch, M.L., Crampton, W.G.R., Davis, J.K., Sullivan, J.P., Lavoue, S., Hopkins, C.D., 2010. Sexual signal evolution outpaces ecological divergence during electric fish species radiation. Am. Nat. 176, 335-356.

Baumgarten, L., Machado-Schiaffino, G., Henning, F., Meyer, A., 2015. What big lips are good for: on the adaptive function of repeatedly evolved hypertrophied lips of cichlid fishes. Biol. J. Linn. Soc. 115, 448-455.

Bejerano, G., Pheasant, M., Makunin, I., Stephen, S., Kent, W.J., Mattick, J.S., Haussler, D., 2004. Ultraconserved elements in the human genome. Science 304, 13211325.

Bolger, A.M., Lohse, M., Usadel, B., 2014. Trimmomatic: A flexible trimmer for Illumina Sequence Data. Bioinformatics, btu170.

Bouckaert, R., Heled, J., Kühnert, D., Vaughan, T., Wu, C.-H., Xie, D., et al., 2014. BEAST 2: A software platform for bayesian evolutionary analysis. PLoS Comput. Biol. 10, e1003537.

Brawand, D. et al., 2014. The genomic substrate for adaptive radiation in African cichlid fish. Nature 513, 375-381.

Bryant, D., Mouton, V., 2004. Neighbor-net: an agglomerative method for the construction of phylogenetic networks. Mol. Biol. Evol. 21, 255-265.

Bryant, D., Bouckaert, R., Felsenstein, J., Rosenberg, N., RoyChoudhury, A., 2012. Inferring species trees directly from biallelic genetic markers: bypassing gene trees in a full coalescent analysis. Molec. Biol. Evol. 19, 1917-1932.

Cowman, P.F., Bellwood, D.R., van Herwerden, L., 2009. Dating the evolutionary origins of wrasse lineages (Labridae) and the rise of trophic novelty on coral reefs. Molec. Phylogen. Evol. 52, 621-631.

Crawford, N.G., Faircloth, B.C., McCormack, J.E., Brumfield, R.T., Winker, K., Glenn, T. C., 2012. More than 1000 ultraconserved elements provide evidence that turtles are the sister group of archosaurs. Biol. Lett. 8, 783-786.

Danecek, P., Auton, A., Abecasis, G., et al., 2011. The variant call format and VCFtools. Bioinformatics 27, 2156-2158.

Danley, P.D., Kocher, T.D., 2001. Speciation in rapidly diverging systems, lessons from Lake Malawi. Mol. Ecol. 10, 1075-1086.

Faircloth, B.C., 2016. PHYLUCE is a software package for the analysis of conserved genomic loci. Bioinformatics 32, 786-788.

Faircloth, B.C., McCormack, J.E., Crawford, N.G., Harvey, M.G., Brumfield, R.T., Glenn, T.C., 2012. Ultraconserved elements anchor thousands of genetic markers spanning multiple evolutionary timescales. Syst. Biol. 61, 717-726.

Faircloth, B.C., Sorenson, L., Santini, F., Alfaro, M.E., 2013. A phylogenomic perspective on the radiation of ray-finned fishes based upon targeted sequencing of ultraconserved elements (UCEs). PLoS One 8, e65923.

Faircloth, B.C., Branstetter, M.G., White, N.D., Brady, S.G., 2014. Target enrichment of ultraconserved elements from arthropods provides a genomic perspective on relationships among Hymenoptera. Mol. Ecol. Res. 15, 489-501.

Felsenstein, J., 2005. PHYLIP (Phylogeny Inference Package) version 3.6.

Fraser, G.J., Hulsey, C.D., Bloomquist, R.F., Uyesugi, K., Manley, N.R., Streelman, J.T., 2009. An ancient gene network is co-opted for teeth on old and new jaws. PLoS Biol. 7, e1000031.

Friedman, M., Keck, B.P., Dornburg, A., Eytan, R.I., Martin, C.H., Hulsey, C.D., Wainwright, P.C., Near, T.J., 2013. Molecular and fossil evidence place the origin of cichlid fishes long after Gondwanan rifting. Proc. Roy. Soc. Ser. B 280, 20131733.

Fryer, G., 1959. The trophic interrelationships and ecology of some littoral communities in Lake Nyasa with especial reference to the fishes, and a discussion of the evolution of a group of rock-frequenting Cichlidae. Proc. Zool. Soc. Lond. 132, 15-281.

Fryer, G., Iles, T.D., 1972. The cichlid fishes of the great lakes of Africa. Oliver and Boyd, Edinburgh.

Gavrilets, S., Losos, J.B., 2009. Adaptive radiation: contrasting theory with data. Science 323, 732-737.

Genner, M.J., Ngatunga, B.P., Mzighani, S., Smith, A., Turner, G.F., 2015. Geographical ancestry of Lake Malawi's cichlid fish diversity. Biol. Lett. 11, 20150232.

Glenn, T.C., Nilsen, R., Kieran, T.J. Finger, J.W. Pierson, T.W., Bentley, K.E., Hoffberg, S., Louha, S., Garcia-De-Leon, F.J., del Rio Portilla, M.A., Reed, K., Anderson, J.L. Meece, J.K., Aggery, S., Rekaya, R., Alabady, M., Belanger, M., Winker, K.,
Faircloth, B.C., 2016. Adapterama I: Universal stubs and primers for thousands of dual-indexed Illumina libraries (iTru \& iNext). Molec. Ecol. Res. (in press).

Glor, R.E., 2010. Phylogenetic insights on adaptive radiation. Ann. Rev. Ecol. Evol. 41, 251-270.

Grabherr, M.G. et al., 2011. Full-length transcriptome assembly from RNA-Seq data without a reference genome. Nat. Biotech. 29, 644-652.

Genner, M.J., Seehausen, O., Cleary, D.F.R., Knight, M.E., Michel, E., Turner, G.F., 2004. How does the taxonomic status of allopatric populations influence species richness within African cichlid fish assemblages? J. Biogeogr. 31, 93-102.

Genner, M.J., Turner, G.F., 2005. The mbuna cichlids of Lake Malawi: a model for rapid speciation and adaptive radiation. Fish. Fisheries. 6, 1-34.

Harmon, L.J., Melville, J., Larson, A., Losos, J.B., 2008. The role of geography and ecological opportunity in the diversification of day geckos (Phelsuma). Syst. Biol. 57, 562-573.

Henning, F., Machado-Schiaffino, G., Baumgarten, L., Meyer, A., 2017. Genetic dissection of adaptive form and function in rapidly-speciating cichlid fishes. Evolution 71, 1297-1312.

Higham, T.E., Birn-Jeffery, A., Collins, C.E., Hulsey, C.D., Russell, A.P., 2015. Adaptive simplification and the evolution of gecko locomotion: Morphological and biomechanical consequences of losing adhesion. Proc. Nat. Acad. Sci. U.S.A. 112, 809-814.

Hodge, J.R., Read, C.I., van Herwerden, L., Bellwood, D.R., 2012. The role of peripheral endemism in species diversification: Evidence from the coral reef fish genus Anampses (Family: Labridae). Molec. Phylogen. Evol. 62. 653-633.

Hollingsworth Jr., P.R., Simons, A.M., Fordyce, J.A., Hulsey, C.D., 2013. Explosive diversification following a benthic to pelagic shift in freshwater fishes. BMC Evol. Biol. 13, 272.

Hulsey, C.D., 2009. Cichlid genomics and phenotypic diversity in a comparative context. Integrat. Comp. Biol. 49, 618-629.

Hulsey, C.D., Mims, M.C., Streelman, J.T., 2007. Do constructional constraints influence cichlid craniofacial diversification? Proc. Roy. Soc. Ser. B 274, 18671875.

Hulsey, C.D., Mims, M.C., Parnell, N.F., Streelman, J.T., 2010. Comparative rates of lower jaw diversification in cichlid adaptive radiations. J. Evol. Biol. 23, 1456-

Hulsey, C.D., Roberts, R.J., Loh, Y.H.E., Rupp, M.F., Streelman, J.T., 2013a. Lake Malawi cichlid evolution along a benthic/limnetic axis. Ecol. Evol. 3, 2262-2272.

Hulsey, C.D., Keck, B.P., Alamillo, H., O'Meara, B.C., 2013b. Mitochondrial genome primers for Lake Malawi cichlids. Molec. Ecol. Res. 13, 347-353.

Huson, D.H., Bryant, D., 2006. Application of phylogenetic networks in evolutionary studies. Mol. Biol. Evol. 23, 254-267.

Husemann, M., Tobler, M., McCauley, C., Ding, B., Danley, P., 2014. Evolution of body shape in differently coloured sympatric congeners and allopatric populations of Lake Malawi's rock-dwelling cichlids. J. Evol. Biol. 27, 826-839.

Ivory, S.J., Blome, M.W., King, J.W., McGlue, M.M., Cole, J.E., Cohen, A.S., 2016. Environmental change explains cichlid adaptive radiation at Lake Malawi over the past 1.2 million years. Proc. Nat. Acad. of Sci. U.S.A. 113, 1189511900.

Joyce, D.A., Lunt, D.H., Genner, M.J., Turner, G.F., Bills, R., Seehausen, O., 2011. Repeated colonization and hybridization in Lake Malawi cichlids. Curr. Biol. 21, R108-R109.

Katoh, K., Kuma, K., Toh, H., Miyata, T., 2005. MAFFT version 5: improvement in accuracy of multiple sequence alignment. Nuc. Acid. Res. 20, 511-518.

Kautt, A.F., Elmer, K.R., Meyer, A., 2012. Genomic signatures of divergent selection and speciation patterns in a natural 'experiment', the young parallel radiations of Nicaraguan crater lake cichlid fishes $21,4770-4786$.

Kautt, A.F., Machado-Schiaffino, G., Torres-Dowdall, J., Meyer, A., 2016. Incipient sympatric speciation in Midas cichlid fish from the youngest and one of the smallest crater lakes in Nicaragua due to differential use of the benthic and limnetic habitats? Ecol. Evol. 6, 5342-5357.

Kiessling, W., Simpson, C., 2011. On the potential for ocean acidification to be a general cause of ancient reef crises. Glob. Change Biol. 17, 56-67.

Kocher, T.D., 2004. Adaptive evolution and explosive speciation: the cichlid fish model. Nat. Rev. Genet. 5, 288-298.

Kocher, T.D., Conroy, J.A., McKaye, K.R., Stauffer, J.R., Lockwood, S.F., 1995. Evolution of NADH dehydrogenase subunit 2 in East African cichlid fish. Mol. Phylog. Evol. $4,420-432$.

Konings, A., 1991. Konings' book of cichlids and all the other fishes of Lake Malawi. TFH Publications, Inc. pp. 448

Lanfear, R., Calcott, B., Ho, S.Y., Guindon, S., 2012. Partitionfinder: Combined 355 selection of partitioning schemes and substitution models for phylogenetic analyses. Mol. Biol. Evol. 29, 1695-1701.

Loh, E.Y.H., Katz, L.S., Mims, M.C., Kocher, T.D. Yi, S.V. Streelman, J.T., 2008. Comparative analysis reveals signatures of differentiation amid genomic polymorphism in Lake Malawi cichlids. Genom. Biol. 9, R113.

López-Fernández, H., Arbour, J.H., Winemiller, K.O., Honeycutt, R.L., 2012. Testing for ancient adaptive radiations in Neotropical cichlid fishes. Evolution 67, 13211337.

MacArthur, R.H., Levins, R., 1964. Competition, habitat selection, and character displacement in a patchy environment. Proc. Natl. Acad. Sci. U.S.A. 51, 12071210.

Malinsky, M., Salzburger, W., 2016. Environmental context for understanding the iconic adaptive radiation of cichlid fishes in Lake Malawi. Proc. Natl Acad. Sci. U. S.A. $113,11654-11656$.

Martin, C.H., Genner, M.J., 2009. High niche overlap between two successfully coexisting pairs of Lake Malawi cichlids. Can. J. Fish. Aquat. Sci. 66, 579-588. 
McCormack, J.E., Faircloth, B.C., Crawford, N.G., Gowaty, P.A., Brumfield, R.T., Glenn, T.C., 2012. Ultraconserved elements are novel phylogenomic markers that resolve placental mammal phylogeny when combined with species tree analysis. Genome. Res. 22, 746-754.

McGee, M.D., Faircloth, B.C., Borstein, S.R., Zheng, J., Hulsey, C.D., Wainwright, P.C., Alfaro, M.E., 2016. Replicated divergence in cichlid radiations mirrors a major vertebrate innovation. Proc. Roy. Soc. Ser. B 283, 20151413.

Meyer, A., 1993. Phylogenetic relationships and evolutionary processes in East African cichlids. T.R.E.E. 8, 279-284.

Meyer, A., 1994. Shortcomings of the cytochrome b gene as a molecular marker. T.R. E.E. 9, 278-280.

Meyer, A., Kocher, T.D., Basasibwaki, P., Wilson, A.C., 1990. Monophyletic origin of Lake Victoria cichlid fishes suggested by mitochondrial DNA sequences. Nature 347, 550-553.

Meyer, A., Montero, C.M., Spreinat, A., 1996. Molecular phylogenetic inferences about the evolutionary history of the East African cichlid fish radiations. In: Johnson, T., Odada, E. (Eds.), IDEAL (International Decade of East African Lakes): The Limnology, Climatology and Paleoclimatology of the East African Lakes. Gordon and Breach Scientific Publishers, New York, NY, pp. 303-323.

Mims, M.C., Hulsey, C.D., Fitzpatrick, B.M., Streelman, J.T., 2010. Geography disentangles introgression from ancestral polymorphism in Lake Malawi cichlids. Mol. Ecol. 19, 940-951.

Machado-Schiffiano, G., Kautt, A.F., Kusche, H., Meyer, A., 2015. Crater lakes as a cradle of divergence in Ugandan cichlid fishes: parallel morphological and genetic differentiation among lakes. BMC Evol. Biol. 15, 9.

Moran, P., Kornfield, I., 1993. Retention of an ancestral polymorphism in the mbuna species flock (Teleostei, Cichlidae) of Lake Malawi. Molec. Biol. Evol. 10, 1015 1029.

Muschick, M., Nosil, P., Roesti, M., Dittmann, M.T., Harmon, L., Salzburger, W., 2014 Testing the stages model in the adaptive radiation of cichlid fishes in East African Lake Tanganyika. Proc. R. Soc. B 281, 20140605.

Parnell, N.F., Streelman, J.T., 2011. The macroecology of rapid evolutionary radiation. Proc. R. Soc. Lond. B 278, 2486-2494.

Price, S.A., Holzman, R., Near, T.J., Wainwright, P.C., 2011. Coral reefs promote the evolution of morphological diversity and ecological novelty in labrid fishes. Ecol. Lett. 14, 462-469.

Price, S.A., Tavera, J.J., Near, T.J., Wainwright, P.C., 2013. Elevated rates o morphological and functional diversification in reef-dwelling haemulid fishes. Evolution 67, 417-428.

Rambaut, A., Suchard, M.A., Xie, D., Drummond, A.J., 2014. Tracer v1.6 http://beast. bio.ed.ac.uk/Tracer.

Reinthal, P.N., 1990. The feeding habits of a group of herbivorous rock-dwelling cichlid fishes (Cichlidae, Perciformes) from Lake Malawi, Africa. Environ. Biol. Fish. 27, 215-233.
Ribbink, A.J., Marsh, B.A., Marsh, A.C., Ribbink, A.C., Sharp, B.J., 1983. A preliminary survey of the cichlid fishes of rocky habitats in Lake Malawi. S. Afric. J. Zool. 18 $149-310$.

Robinson, B.W., Wilson, D.S., 1998. Optimal foraging, specialization, and a solution to Liem's paradox. Am. Nat. 151, 223-235.

Rohland, N., Reich, D., 2012. Cost-effective, high-throughput DNA sequencing ibraries for multi-plexed target capture. Genome Res. 22, 939-946.

Rupp, M.F., Hulsey, C.D., 2014. Influence of substrate orientation on feeding kinematics and performance of algae grazing Lake Malawi cichlid fishes. J. Exp. Biol. 217, 3057-3066.

Sallan, L.C., Friedman, M., 2012. Heads or tails: staged diversification in vertebrate evolutionary radiations. Proc. R. Soc. B 279, 2025-2032.

Salzburger, W., Van Bocxlaer, B., Cohen, A.S., 2014. Ecology and evolution of the African great lakes and their faunas. Annu. Rev. Ecol. Evol. Syst. 45, 519-545.

Salzburger, W., 2009. The interaction of sexually and naturally selected traits in the adaptive radiations of cichlid fishes. Molec. Ecol. 18, 169-185.

Santos-Santos, J.H., Audenaert, L., Verheyen, E., Adriaens, D., 2015. Divergent ontogenies of trophic morphology in two closely related haplochromine cichlids. J. Morph. 276, 860-871.

Schluter, D., 2000. The Ecology of Adaptive Radiation. Oxford University Press, Oxford.

Schoener, T.W., 1974. Resource partitioning in ecological communities. Science 185 27-39.

Shaw, P.W., Turner, G.F., Idid, M.R., Robinson, R.L., Carvalho, G.R., 2000. Genetic population structure indicates sympatric speciation of Lake Malawi pelagic cichlids. Proc. Roy. Soc. Ser. B 267, 2273-2280.

Sidlauskas, B., 2008. Continuous and arrested morphological diversification in sister clades of Characiform fishes: a phylomorphospace approach. Evolution 62 3135-3156.

Stamatakis, A., 2014. RAxML version 8: a tool for phylogenetic analysis and postanalysis of large phylogenies. Bioinformatics 30, 1312-1313.

Streelman, J.T., Danley, P.D., 2003. The stages of vertebrate evolutionary radiation. T. R.E.E. 18, 126-131.

Tornabene, L., Valdez, S., Erdmann, M., Pezold, F., 2015. Support for a 'Center of Origin' in the Coral Triangle: Cryptic diversity, recent speciation, and local endemism in a diverse lineage of reef fishes (Gobiidae: Eviota). Molec Phylogen. Evol. 82, 200-212.

Won, Y.J., Sivasundar, A., Wang, Y., Hey, J., 2005. On the origin of Lake Malawi cichlid species: a population genetic analysis of divergence. Proc. Nat. Acad. Sci. U.S.A. $102,6581-6586$.

York, R.A., Patil, C., Hulsey, C.D., Streelman, J.T., Fernald, R.D., 2015. Evolution of bower building in Lake Malawi cichlid fish: phylogeny, morphology, and behavior. Front. Ecol. Evol. 3, 18. 